\title{
Identification of Nontuberculous Mycobacteria Isolated From Household Showerheads of Patients With Nontuberculous Mycobacteria
}

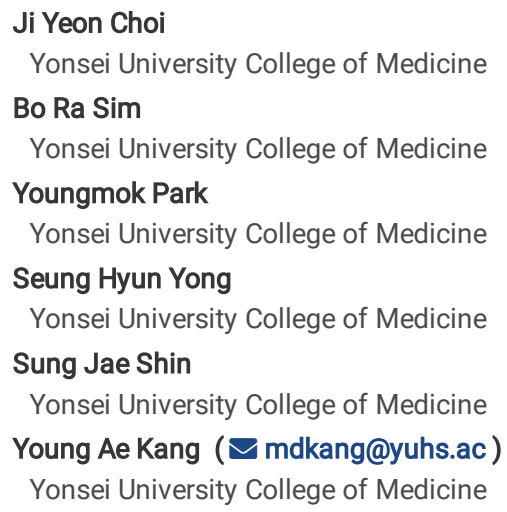

Research Article

Keywords: Nontuberculous mycobacteria, Environmental source, Multiplex PCR, Showerhead

Posted Date: February 8th, 2022

DOI: https://doi.org/10.21203/rs.3.rs-1248306/v1

License: (c) (1) This work is licensed under a Creative Commons Attribution 4.0 International License. Read Full License 


\section{Abstract}

This study aimed to examine whether nontuberculous mycobacteria (NTM) inside household showerheads are identical to those in patients with NTMpulmonary disease (PD) since household water is one of the potential NTM sources. Samples were obtained from 32 household showerheads of patients with NTM-PD recruited through the Pulmonary Outpatient Department at the Severance Hospital between October 2018 and October 2019 . All isolates from patients with NTM-PD were diagnosed using a reverse-hybridization line probe assay based on the rop $B$ gene. To determine the mycobacterial compositions, the washing fluids were collected and investigated using multiplex polymerase chain reaction assay and NTM culture; suspected microbial isolates in these fluids and culture were identified using sequencing analysis of 16S rRNA gene. NTM species causing the PD in the patients were Mycobacterium avium, $M$. intracellulare, $M$. abscessus, $M$. massiliense, and $M$. fortuitum complex. The mycobacteria isolated from the showerhead were M. lentiflavum, $M$. gordonae, $M$. triplex, M. phocaicum, M. mucogenicum, M. florentinum, M. gilvum, M. Ilatzerense, and M. peregrinum. However, the species identified in the showerheads did not match those of the patients. Despite NTM species in the showerheads, clinical implications in the main pathogenesis associated with the disease in the patients studied were not elucidated.

\section{Introduction}

Nontuberculous mycobacteria (NTM) species are opportunistic pathogens responsible for progressive pulmonary disease as well as skin and soft tissue infection, lymphadenitis, and other health issues ${ }^{1}$. An increase in the incidence and prevalence of pulmonary disease caused by NTM has been reported worldwide ${ }^{2}$. In Korea, the incidence and prevalence of NTM infection increased rapidly from 2003 to 2016, particularly among women and older age groups. For reported cases of NTM infection, the incidence and prevalence rates were 17.9 and 33.3 per 100,000 populations in 2016, respectively. Additionally, the mortality rate in the NTM infected-population was higher than that in the general population ${ }^{3}$.

M. avium complex (MAC) members, such as $M$. avium and $M$. intracellulare; $M$. abscessus complex (MABC) members, such as $M$. abscessus and $M$. massiliense are the major causative organisms of NTM pulmonary disease and are frequently isolated from patients with NTM pulmonary disease. Recent studies have focused on identifying risk factors for both host and environmental factors associated with NTM pulmonary disease. NTM has been isolated from different environmental sources, including water, soil, food, dust, and aerosols ${ }^{4,5}$. Infections with NTM are caused by ingestion or inhalation of contaminated food or aerosol, and through injured skin, which indicates that a significant entry gate of NTM into a host organism is from environmental sources, such as water ${ }^{6}$. Previous studies have frequently identified mycobacteria in household water systems, and it has raised considerable concern among individuals susceptible to this infection. Some studies reported that patients with chronic obstructive pulmonary disease or immunodeficiency could have developed acute disorder when exposed to water contaminated with $\mathrm{NTM}^{7,8}$. To date this evidence has been reported mainly for MAC ${ }^{4,9-13}$. Thomson et al. showed that disease-causing NTM species were isolated from household water and aerosols, such as M. avium, M. kansasii, M. lentiflavum, and M. abscessus $^{14}$. Although implicated as a potential source of disease, the mycobacterial composition and environmental predictors of showerhead-associated mycobacteria remain unresolved. Accordingly, there is a need for further epidemiological investigations of potential sources of NTM infections, including showerheads. Our study aimed to isolate and identify the NTM species present in household showerheads and compare the NTM found in biofilm with the NTM from patients with NTM pulmonary disease.

\section{Methods}

\section{Sample collection and mycobacteria isolation from household showerheads}

Patients with NTM-PD were recruited through the Pulmonary Outpatient Department at the Severance Hospital between October 2018 and October 2019 . All patients with NTM-PD were diagnosed according to the American Thoracic Society/Infectious Disease Society of America (ATS/IDSA) 2007 guidelines ${ }^{15}$. For AFB smear and cultures were examined by fluorochrome staining using auramine-rhodamine and culturing in $3 \%$ Ogawa medium and mycobacteria growthindicator tube medium (MGIT; Becton Dickson, NJ, USA). A reverse-hybridization line probe assay based on the rpoB gene, conducted at Seoul Clinical Laboratories (Yongin, Korea), was used for NTM species identification.

Following analysis of NTM, showerheads were collected from the homes of the patients with NTM, and a total of 32 samples were collected and 7 of the 32 samples included the shower hose. After disassembling the showerhead, the inside was wiped with a cotton swab, and the cotton swab was put in $50 \mathrm{~mL}$ of phosphate-buffered saline (PBS) and vortexed for $1 \mathrm{~min}$. In the case of shower hoses, the front and rear entrances were parafilmed after adding $0.05 \%$ tween 20 in PBS into the shower hose. Then, the shower hose was sealed in a sterilized plastic bag and sonicated in a water bath for 5 min. After vortexing or sonicating, microbial cells in PBS were pelleted by centrifugation at 4,000 rpm for $30 \mathrm{~min}$. After centrifugation, the supernatant was removed, and the pellet was resuspended in $3 \mathrm{~mL}$ of PBS. The sample collection process from showerheads is briefly presented in Supplemental Figure 1. The resuspended pellets were stored at $4^{\circ} \mathrm{C}$ before further use. To identify the mycobacterium from natural samples, the analysis was performed using resuspended samples and cultivated of the isolates. Additionally, all resuspended samples $(1 \mathrm{~mL})$ were stained by Ziehl-Neelsen staining, and smear-positivity was confirmed by light microscope at $100 \times$ magnification $^{16}$.

\section{Supplementation of media and growth conditions}

For cultivation, the resuspended samples were decontaminated with $0.01 \%$ cetylpyridinium chloride (CPC, Sigma) and cultivated in Mycobacteria Growth Indicator Tubes (MGIT, BD BACTEC). They were supplemented with PANTA/enrichment (BD BACTEC) to obtain the following final concentrations of antibiotics in the culture medium: $40 \mathrm{U} / \mathrm{mL}$ of polymyxin, $4 \mu / \mathrm{mL}$ of amphotericin $\mathrm{B}, 16 \mu / \mathrm{mL}$ of nalidixic acid, $4 \mu / \mathrm{mL}$ of trimethoprim, and $4 \mu / \mathrm{mL}$ of azlocillin for 4 weeks (slow growers) in the MGIT system. After 4 weeks, the samples were subcultured onto $7 \mathrm{H} 11$ agar supplemented with $10 \%$ oleic acid-albumin-dextrose- 
catalase (OADC, Difco) for 4 weeks at $37^{\circ} \mathrm{C}$. After 4 weeks, they were subcultured onto Ogawa medium for 4 weeks at $37^{\circ} \mathrm{C}^{17}$. Colonies of putative acid-fast bacteria were picked after $5,10,21$, and 28 days.

\section{DNA preparation, multiplex polymerase chain reaction (PCR), and identification of mycobacterial target sequences}

DNA was extracted from $1 \mathrm{~mL}$ of the resuspended sample using the conventional cetyltrimethylammonium bromide method (CTAB) as previously described ${ }^{18}$. Three primers were used in the PCR (IS 1311, DT1, and 16s rRNA) according to a previously described method (Table 1) ${ }^{19,20}$. For amplification, each PCR mixture contained $25 \mu \mathrm{L}$ of $2 X$ EF-Taq PCR Smart mix (Solgent Co., Ltd. Daejeon, South Korea), $2 \mu \mathrm{L}$ each of the 4 primer sets (all primer solutions in 10 pmol), $2 \mu \mathrm{L}$ of DNA template, and $17 \mu \mathrm{L}$ of water in a final volume of $50 \mu \mathrm{L}$. PCR was performed at $61.5^{\circ} \mathrm{C}$ for $45 \mathrm{~s}$ for the annealing step, and followed by 30 cycles. The rest of the PCR parameters and electrophoresis were performed based on previous publications ${ }^{19}$. According to the multiplex PCR interpretation criteria, amplification of $16 \mathrm{~S}$ rRNA was interpreted as identification of $M$. tuberculosis, amplification of IS 1311 or DT1 with the 16S rRNA gene was interpreted as identification of $M$. avium or M. intracellulare, respectively. Mycobacterium species outside the multiplex PCR target species were indicated by observation of only $16 \mathrm{~S}$ rRNA gene amplification ${ }^{19}$.

Isolates identified as other Mycobacterium spp. were further determined using 16S rRNA sequencing in liquid and colony samples. Sequencing reactions were performed using the BigDye (R) Terminator v3.1 Cycle Sequencing Kit from Applied Biosystems. At the end of the reaction, the dNTPs and the reactants that were not involved in the response were removed by the method recommended by Applied Biosystems. Then, the samples were loaded onto $A B I$ Prism $3730 X L$ DNA Analyzer to obtain sequencing results. The analyzed sequences were determined using the Sequencher 5.4 .6 program from Gene Codes. The base sequence was searched in BLAST.

\section{Ethics approval}

This research protocol was approved by the Institutional Review Board of the Severance Hospital, South Korea (IRB No. 4-2018-0444), and the study design was approved by the appropriate ethics review boards. All methods were carried out in accordance with the approved guidelines and regulations as well as in accordance with the Declaration of Helsinki. All patients gave their written informed consent.

\section{Results}

\section{Baseline characteristics}

A total of 32 patients were diagnosed with NTM pulmonary disease before the study period. The baseline characteristics of the patients are summarized in Table 2. There were 25 (78\%) female patients, and the median age of all patients was 59 years (range 30 to 72 years). Seventeen (53\%) had Mycobacterium avium infection, seven (22\%) had $M$. intracellulare infection, and one each (3\%) had $M$. abscessus, M. massiliense, and $M$. fortuitum complex infections. A further $5(16 \%)$ had mixed infections. Ten of these patients were naïve for NTM treatment and the others had a history of previous or ongoing treatment for NTM lung disease. Eight (25\%) patients had a history of prior treatment for pulmonary tuberculosis. The median prevalence period of NTM was 20 years (range 0.1 to 11.2 years). Up to 6 months prior to enrollment in this study, 30 patients were acid-fast bacillus (AFB) positive in sputum smears.

\section{Isolation of NTM from the washing fluids of showerheads using multiplex PCR}

As described in Methods and Materials, samples were obtained by a swab of the surfaces of 32 showerheads, including the shower hoses from seven of the 32 showers. To isolate the NTM from the showerheads, we amplified rRNA genes from resuspended sample DNAs by PCR, using three primers (IS 1311, DT1, and 16s rRNA, Table 1). Figure 1 shows the amplification products of isolates using multiplex PCR primer sets. Seventeen samples showed a single amplification band of 500 base pair (bp) product specific to the 16S rRNA gene for all mycobacteria. The sizes of the resulting PCR products and the groups that they identify are described in Table 1 . In addition, the AFB smear was performed using resuspended samples, and identified positive results in eight samples $(1+, n=7 ; 4+, n=1$; negative, $n=16$, and non-detected, $n=8$; Table 3 ).

\section{Identification of NTM in both liquid and culture samples from the showerheads by genetic analysis}

In order to identify accurate mycobacterium spp., genotype sequencing was performed in liquid and colonies of isolates sampled by PCR assay using $16 \mathrm{~s}$ rRNA and rpoB. The species of NTM isolated from washing fluids and colonies from 32 showerheads included $M$. florentinum, M. phocaicum, M. gordonae, M. mucogenicum, M. gilvum, M. Ilatzerense, M. peregrinum, M. sherrisii, M. aurum, M. porcinum, M. lentiflavum, M. colombiense, M. triplex, M. gordonae, and M. arupense, and details are summarized in Table 3. However, none of the species from the showerhead washing fluids and cultivating colonies matched the NTM isolates from patients with NTM pulmonary disease.

\section{Discussion}

In light of the increasing prevalence of NTM pulmonary disease without a known cause, clinicians have a substantial interest in identifying the source of the NTM infection and the number of species identified in both clinical samples and environments. In this study, NTM was isolated from the showerheads as a potential source of infection for patients with NTM-PD, and a total of 18 species (subspecies, complexes) of NTM were identified. Among them, none of the mycobacteria matched the patients' infectious mycobacteria, but various NTM species were identified, suggesting that it could be a potential source of infection in susceptible persons.

NTM are ubiquitous in the environment and have been isolated from different environmental sources, particularly water in homes, swimming pools, whirlpool therapy baths, soils, and the workplace 21,22 . Geographic and behavioral variabilities are also associated with the incidence of NTM infection among 
patients ${ }^{23}$. Particularly, water is one of the main vehicles for NTM transmission, as confirmed by isolation of the same species from both water and patients in several studies. For example, Covert et al. reported that $35 \%$ of samples from municipal water supplies in 21 states in the United States were found to test positive for $\mathrm{NTM}^{24}$. In one study, strains from the water system identical to those in the patients were found in 7 (41\%) of 17 patients sampled ${ }^{12}$. For another example, Feazel et al. showed that the showerhead environment strongly enriched for microbes that are known to form biofilms in water systems, including Mycobacterium spp., Shpingomonas spp., Methylobacterium spp. and others in United States ${ }^{25}$. Our results, and those described by various studies, show that NTM are quite frequent in household water. However, in this study, we rarely detected the major causative organisms of NTM disease, including MAC members, such as $M$. avium and $M$. intracellulare, or $M$. abscessus, M. massiliense from the surfaces of the showerheads. In addition, when the isolates from the patients' showerheads were compared with those from their sputum samples, none had matching pathogens in the present study. Many factors affect the ability of NTM to adhere and form a biofilm on various materials ${ }^{26}$. The materials used for the showerhead, the water sources, and the type of hot-water supply system may influence the regional presence of NTM spp. on showerheads. In addition to exposure, host factors are influential in NTM infection ${ }^{7,8,27-29}$. According to the study by Falkinham et al. ${ }^{30}$, the potential impact of human activities on the ecology of NTM has also been described. Our study examined the household showerhead as a potential source of NTM. Although we did not demonstrate Mycobacteria spp., which was the main pathogen associated with disease in the patients diagnosed with NTM infection, we found that 17 (57\%) biofilm (swab) samples collected from the showerheads of patients with NTM pulmonary disease yielded NTM, using 16S rRNA sequences. In this study, many of the isolates were identified as M. lentiflavum 7 (10.9\%), M. gordonae 6 (9.4\%), M. triplex 4 (6.3\%), and M. mucogenicum 3 (4.7\%). In our study, as in the results of several studies, M. lentiflavum was also the most frequently identified isolate from the house water system and could be a potential pathogen that can cause pulmonary lung disease ${ }^{14,31,32}$.

M. gordonae is known to rarely causes pulmonary lung disease due to its low pathogenicity, and when M. gordonae is detected in sputum, it has been considered as a contamination ${ }^{33}$. However, there are case reports that $M$. gordonae can cause pulmonary lung disease in immunosuppressed patients such as human immunodeficiency virus infection, steroid treatment, and organ transplant patients ${ }^{34-36}$. Moreover, $M$. triplex and $M$. mucogenicum be known as opportunistic infections in both immunocompromised and immunocompetent humans exposed to environmental sources and may be fatal if the infection is disseminated ${ }^{37-39}$. Accordingly, our study supports the evidence that a common indoor shower facility can be a potential source of NTM infection, which has clear relevance to public health.

The limitation of this study is that we did not consider some relevant factors, such as household water sources, estimated time since showerhead installation, usage frequency, and cleaning frequency, which may influence the ability of NTM to adhere and form a biofilm. The strength of this study is that we recovered the NTM from environmental samples by establishing methods of sample collection, processing, and cultivation.

In conclusion, NTM are widely distributed in the environment and people are exposed to it frequently. There are several known vehicles of NTM transmission, of which water is one of the most important ones. In the present study, despite not consistent with the patients' infectious mycobacteria, we showed that the showerhead could be a potential source of NTM infection by identifying NTM through isolation and cultivation. In combination with the earlier studies, these findings will help to elucidate the infectious source and route of NTM-PD.

\section{Declarations}

\section{Authors' Contributions}

C.J.Y. and S. B.R. designed the report and wrote the paper; P.Y.M., Y.S.H., and S.S.J. drafted and revised the manuscript; K.Y.A. designed the concept and finally approved the paper. All authors have taken due care to ensure the integrity of this work, and this final manuscript has been seen and approved by all authors.

\section{Acknowledgments}

None.

\section{Data availability statement}

All data generated or analysed during this study are included in this published article and its supplementary information files.

\section{Competing Interests Statement}

The authors declare that they have no competing interests.

\section{References}

1. Piersimoni, C. \& Scarparo, C. Extrapulmonary infections associated with nontuberculous mycobacteria in immunocompetent persons. Emerging infectious diseases 15, 1351 (2009).

2. Winthrop, K. L. et al. Incidence and prevalence of nontuberculous mycobacterial lung disease in a large US managed care health plan, 2008-2015. Annals of the American Thoracic Society 17, 178-185 (2020).

3. Park, S. C. et al. Prevalence, incidence, and mortality of nontuberculous mycobacterial infection in Korea: a nationwide population-based study. BMC pulmonary medicine 19, 1-9 (2019).

4. Falkinham III, J. O., Iseman, M. D., de Haas, P. \& van Soolingen, D. Mycobacterium avium in a shower linked to pulmonary disease. Journal of water and health 6, 209-213 (2008). 
5. De Groote, M. A., Pace, N. R., Fulton, K. \& Falkinham, J. O. Relationships between Mycobacterium isolates from patients with pulmonary mycobacterial infection and potting soils. Applied and environmental microbiology 72, 7602-7606 (2006).

6. Falkinham 3rd, J. Epidemiology of infection by nontuberculous mycobacteria. Clinical microbiology reviews 9, 177 (1996).

7. Marras, T. K. \& Daley, C. L. Epidemiology of human pulmonary infection with nontuberculous mycobacteria. Clinics in chest medicine 23, $553-568$ (2002).

8. Kim, J. S. et al. Nontuberculous mycobacterial infection: CT scan findings, genotype, and treatment responsiveness. Chest 128, 3863-3869 (2005).

9. Castillo-Rodal, A. et al. Potentially pathogenic nontuberculous mycobacteria found in aquatic systems. Analysis from a reclaimed water and water distribution system in Mexico City. European Journal of Clinical Microbiology \& Infectious Diseases 31, 683-694 (2012).

10. Falkinham, J. O. Environmental sources of nontuberculous mycobacteria. Clinics in chest medicine 36, 35-41 (2015).

11. Embil, J. et al. Pulmonary illness associated with exposure to Mycobacterium-avium complex in hot tub water: hypersensitivity pneumonitis or infection? Chest 111, 813-816 (1997).

12. Falkinham III, J. O. Nontuberculous mycobacteria from household plumbing of patients with nontuberculous mycobacteria disease. Emerging infectious diseases 17, 419 (2011).

13. Nishiuchi, Y. et al. The recovery of Mycobacterium avium-intracellulare complex (MAC) from the residential bathrooms of patients with pulmonary MAC. Clinical Infectious Diseases 45, 347-351 (2007).

14. Thomson, R. et al. Isolation of nontuberculous mycobacteria (NTM) from household water and shower aerosols in patients with pulmonary disease caused by NTM. Journal of clinical microbiology 51, 3006-3011 (2013).

15. Society, A. T. An official ATS/IDSA statement: diagnosis, treatment, and prevention of nontuberculous mycobacterial diseases. Am J Respir Crit Care Med. 175, 367-416 (2007)

16. Chen, P. et al. A highly efficient Ziehl-Neelsen stain: identifying de novo intracellular Mycobacterium tuberculosis and improving detection of extracellular M. tuberculosis in cerebrospinal fluid. Journal of clinical microbiology 50, 1166-1170 (2012).

17. Radomski, N. et al. Comparison of culture methods for isolation of nontuberculous mycobacteria from surface waters. Applied and environmental microbiology 76, 3514-3520 (2010).

18. Wilson, K. Preparation of genomic DNA from bacteria. Current protocols in molecular biology 56, 2.4. 1-2.4. 5 (2001).

19. Chae, H. et al. Development of a one-step multiplex PCR assay for differential detection of major Mycobacterium species. Journal of clinical microbiology 55, 2736-2751 (2017).

20. Lee, H., Park, H.-J., Cho, S.-N., Bai, G.-H. \& Kim, S.-J. Species Identification of Mycobacteria by PCR-Restriction Fragment Length Polymorphism of therpoB Gene. Journal of clinical microbiology 38, 2966-2971 (2000).

21. Thomson, R. M. Changing epidemiology of pulmonary nontuberculous mycobacteria infections. Emerging infectious diseases 16,1576 (2010).

22. Cook, J. L. Nontuberculous mycobacteria: opportunistic environmental pathogens for predisposed hosts. British medical bulletin 96, 45-59 (2010).

23. Prevots, D. R., Adjemian, J., Fernandez, A. G., Knowles, M. R. \& Olivier, K. N. Environmental risks for nontuberculous mycobacteria. Individual exposures and climatic factors in the cystic fibrosis population. Annals of the American Thoracic Society 11, 1032-1038 (2014).

24. Covert, T. C., Rodgers, M. R., Reyes, A. L. \& Stelma, G. N. Occurrence of nontuberculous mycobacteria in environmental samples. Applied and environmental microbiology 65, 2492-2496 (1999).

25. Feazel, L. M. et al. Opportunistic pathogens enriched in showerhead biofilms. Proceedings of the National Academy of Sciences 106, 16393-16399 (2009).

26. Mullis, S. N. \& Falkinham III, J. Adherence and biofilm formation of M ycobacterium avium, M ycobacterium intracellulare and M ycobacterium abscessus to household plumbing materials. Journal of applied microbiology 115, 908-914 (2013).

27. Wolinsky, E. Nontuberculous mycobacteria and associated diseases. American Review of Respiratory Disease 119, 107-159 (1979).

28. Olivier, K. N. et al. Nontuberculous mycobacteria: I: multicenter prevalence study in cystic fibrosis. American journal of respiratory and critical care medicine 167, 828-834 (2003).

29. Chan, E. D. et al. Alpha-1-antitrypsin (AAT) anomalies are associated with lung disease due to rapidly growing mycobacteria and AAT inhibits Mycobacterium abscessus infection of macrophages. Scandinavian journal of infectious diseases 39, 690-696 (2007).

30. Falkinham, J. O. Impact of human activities on the ecology of nontuberculous mycobacteria. Future microbiology 5, 951-960 (2010).

31. Lee, E.-S., Lee, M.-Y., Han, S.-H. \& Ka, J.-O. Occurrence and molecular differentiation of environmental mycobacteria in surface waters. Journal of microbiology and biotechnology 18, 1207-1215 (2008).

32. Marshall, H. M. et al. Mycobacterium lentiflavum in drinking water supplies, Australia. Emerging infectious diseases 17, 395 (2011).

33. Griffith, D. E. et al. An official ATS/IDSA statement: diagnosis, treatment, and prevention of nontuberculous mycobacterial diseases. American journal of respiratory and critical care medicine $175,367-416$ (2007).

34. Weinberger, M., Berg, S. L., Feuerstein, I. M., Pizzo, P. A. \& Witebsky, F. G. Disseminated infection with Mycobacterium gordonae: report of a case and critical review of the literature. Clinical infectious diseases 14, 1229-1239 (1992).

35. Mazumder, S. A., Hicks, A. \& Norwood, J. Mycobacterium gordonae pulmonary infection in an immunocompetent adult. North American journal of medical sciences 2, 205 (2010).

36. Choi, J. S. et al. A Case of Mycobacterium gordonae Pulmonary Disease in a Patient with Ulcerative Colitis Treated with Infliximab. Korean Journal of Medicine 89, 452-456 (2015). 
37. Shirata, M., Tamaru, A., Marumo, S. \& Fukui, M. Mycobacterium triplex pulmonary disease in an immunocompetent host: a case report and literature review. IDCases 18, e00648 (2019).

38. Johnson, M. M. \& Odell, J. A. Nontuberculous mycobacterial pulmonary infections. Journal of thoracic disease 6, $210(2014)$.

39. Covert, T. C., Rodgers, M. R., Reyes, A. L. \& Stelma Jr, G. N. Occurrence of nontuberculous mycobacteria in environmental samples. Applied and environmental microbiology 65, 2492-2496 (1999).

\section{Tables}

Table 1. Primers used in the multiplex polymerase chain reaction (PCR) assay according to target Mycobacterium species

\begin{tabular}{|c|c|c|c|c|}
\hline Set & Genetic target & Primer sequences (forward and reverse) & Target organism(s) & Expected product size (bp) \\
\hline \multirow[t]{2}{*}{1} & 16S rRNA & 5' GAGATACTCGAGTGGCGAAC 3' & All mycobacterial species & 506 \\
\hline & & 5' CAACGCGACAAACCACCTAC 3' & & \\
\hline \multirow[t]{2}{*}{2} & IS 1311 & 5' TCGATCAGTGCTTGTTCGCG 3' & M. avium complex & 600 \\
\hline & & 5' CGATGGTGTCGAGTTGCTCT 3' & & \\
\hline \multirow[t]{2}{*}{3} & DT1 & 5' AAGGTGAGCCCAGCTTTGAACTCCA 3' & M. intracellulare & 106 \\
\hline & & 5' GCGCTTCATTCGCGATCATCAGGTG 3' & & \\
\hline \multirow[t]{2}{*}{4} & rрoB & 5' TCAAGGAGAAGCGCTACGA 3' & All mycobacterial species & 360 \\
\hline & & 5' GGATGTTGATCAGGGTCTGC 3' & & \\
\hline
\end{tabular}

Primer sets 1 to 4 were used for the multiplex PCR assay.

Table 2. Clinical characteristics of patients with nontuberculous mycobacteria

\begin{tabular}{ll} 
Clinical characteristics & Values \\
\hline Age, years & $59(30-72)$ \\
\hline Female & $25(78)$ \\
\hline NTM pathogens & \\
\hline M. avium & $17(53)$ \\
\hline M. intracellulare & $7(22)$ \\
\hline M. abscessus & $1(3)$ \\
\hline M. massiliense & $1(3)$ \\
\hline M. fortuitum complex & $1(3)$ \\
\hline Mixed & $5(16)$ \\
\hline No. of positive culture (before 6 months) & $30(93)$ \\
\hline NTM treatment status & \\
\hline Naïve & $10(31)$ \\
\hline On therapy & $8(25)$ \\
\hline Off therapy & $14(44)$ \\
\hline NTM prevalence period, years & $20(1.68-5.80)$ \\
\hline Comorbidities & $1(3)$ \\
\hline Previous pulmonary tuberculosis & $7(22)$ \\
\hline Bronchiectasis & $24(75)$ \\
\hline Chronic obstructive pulmonary disease & $3(9)$ \\
\hline Diabetes mellitus & $1(3)$ \\
\hline Autoimmune diseases & $13)$ \\
\hline Cancer & \\
\hline
\end{tabular}

Values are presented as number of subjects and percentage (\%) or as median (interquartile ranges). 
Mixed, Two of M. avium and M.fortuitum complex, M.avium and M.abscessus, M.avium and M. Massiliense; M.avium, M.intracellulare, M.abscessus, M.Massiliense, and M.fortuitum complex.

Table 3. Identification of clinical isolates by the multiplex polymerase chain reaction (PCR) assay and genotyping in both liquid and culture samples of each subject 
No.

\begin{tabular}{lllll} 
No. & & $\begin{array}{l}\text { 16s } \\
\text { rRNA }\end{array}$ & IS1311 & DT1 \\
\hline 1 & ND & - & - & - \\
\hline 2 & ND & - & - & - \\
\hline 3 & ND & - & - & - \\
\hline 4 & ND & + & - & - \\
\hline 5 & ND & + & - & - \\
\hline
\end{tabular}

16s rRNA Identities

ND ND

ND ND

ND ND

M. triplex $\quad 98 \%$

Non-

Mycobacteria

$7 \quad \mathrm{ND}$

9

\begin{tabular}{cccccc}
11 & - & + & - & - & \\
12 & $1+$ & + & - & - & \\
13 & $4+$ & + & - & - & low \\
\hline 14 & & & &
\end{tabular}

\begin{tabular}{llllll}
\hline 14 & - & - & - & - & $M$ \\
15 & - & + & - & -
\end{tabular}

$\begin{array}{rrrrr}15 & - & + & - & - \\ 16 & - & + & - & -\end{array}$

\begin{tabular}{llllll}
\hline 17 & - & - & - & - & \\
18 & - & + & - & - & \\
19 & $1+$ & + & - & -
\end{tabular}

$\begin{array}{lllll}20 & 1+ & - & - & \end{array}$

\begin{tabular}{|c|c|c|c|c|c|c|c|c|c|c|c|}
\hline 21 & - & - & - & - & M. gordonae & $98 \%$ & low signal & $\begin{array}{l}\text { low } \\
\text { signal }\end{array}$ & MIX & MIX & $\begin{array}{l}M \\
\mathrm{sF}\end{array}$ \\
\hline 22 & - & - & - & - & M. triplex & $99 \%$ & M. gilvum & $98 \%$ & $\begin{array}{l}\text { M. simiae } \\
\text { complex }\end{array}$ & $91 \%$ & $\begin{array}{l}N_{t} \\
M\end{array}$ \\
\hline 23 & $1+$ & - & - & - & M. triplex & $97 \%$ & M. sherrisii & $97 \%$ & $\begin{array}{l}\text { M. } \\
\text { lentiflavum }\end{array}$ & $92 \%$ & $\begin{array}{l}M \\
\text { sF }\end{array}$ \\
\hline 24 & - & + & - & - & M. triplex & $97 \%$ & M. arupense & $98 \%$ & $\begin{array}{l}\text { M. } \\
\text { lentiflavum }\end{array}$ & $99 \%$ & $\begin{array}{l}M \\
c c\end{array}$ \\
\hline 25 & - & + & - & - & M. Ilatzerense & $99 \%$ & M. phocaicum & $99 \%$ & M. fortuitum & $97 \%$ & $\begin{array}{l}M \\
\mathrm{sF}\end{array}$ \\
\hline 26 & - & - & - & - & $\begin{array}{l}\text { M. } \\
\text { florentinum }\end{array}$ & $97 \%$ & $\begin{array}{l}\text { M. } \\
\text { mucogenicum }\end{array}$ & $97 \%$ & $\begin{array}{l}\text { Non- } \\
\text { Mycobacteria }\end{array}$ & $\begin{array}{l}\text { Corynebacterium } \\
\text { sp. }\end{array}$ & lo \\
\hline 27 & $1+$ & - & - & - & low signal & low signal & M. aurum & $97 \%$ & MIX & MIX & lo \\
\hline 28 & - & + & - & - & $\begin{array}{l}\text { M. } \\
\text { florentinum }\end{array}$ & $99 \%$ & M. porcinum & $98 \%$ & MIX & MIX & 10 \\
\hline 29 & $1+$ & - & - & - & M. gordonae & $97 \%$ & Not alignment & $\begin{array}{l}\text { Not } \\
\text { alignment }\end{array}$ & ND & ND & $\mathrm{NI}$ \\
\hline 30 & - & + & - & - & $\begin{array}{l}\text { M. } \\
\text { mucogenicum }\end{array}$ & $98 \%$ & M. porcinum & $97 \%$ & $\begin{array}{l}\text { M. } \\
\text { lentiflavum }\end{array}$ & $98 \%$ & M \\
\hline 31 & - & + & - & - & M. & $98 \%$ & M. porcinum & $97 \%$ & M. & $99 \%$ & $\mathrm{Ni}$ \\
\hline
\end{tabular}

Page 8/9 
ND, not done. Test not performed if the sample is contaminated or is not sufficient.

\section{Figures}

\section{Figure 1}

Agarose gel electrophoresis of polymerase chain reaction (PCR) amplification products using multiplex PCR primer sets on resuspended samples. Only one 500-bp band specific to the 16S rRNA gene was detected in a total of 17samples. Each line is the showerhead sample from each subject. Lane M, molecular size marker (100-bp ladder).

\section{Supplementary Files}

This is a list of supplementary files associated with this preprint. Click to download.

- Analysissequencerawdata.xlsx

- Supplementaryfigure.docx 